\title{
Automatic Diameter Control System Applied to the Laser Heated Pedestal Growth Technique
}

\author{
M.R.B. Andreeta*, L.C. Caraschi, A.C. Hernandes \\ Universidade de São Paulo, Instituto de Física de São Carlos \\ C.P. 369, 13560-970 São Carlos - SP, Brazil
}

Received: July 25, 2002; Revised: December 05, 2002

\begin{abstract}
We described an automatic diameter control system (ADC), for the laser heated pedestal growth technique, that reduces the diameter fluctuations in oxide fibers grown from unreacted and nonsinterized pedestals, to less than $2 \%$ of the average fiber diameter, and diminishes the average diameter fluctuation, over the entire length of the fiber, to less than $1 \%$. The ADC apparatus is based on an artificial vision system that controls the pulling speed and the height of the molten zone within a precision of $30 \mu \mathrm{m}$. We also show that this system can be used for periodic in situ axial doping the fiber. Pure and $\mathrm{Cr}^{3+}$ doped $\mathrm{LaAlO}_{3}$ and pure $\mathrm{LiNbO}_{3}$ were used as model materials.
\end{abstract}

Keywords: $L H P G$, single crystal fiber, diameter control, crystal growth

\section{Introduction}

Single crystal fibers (SCFs) are candidates to be used in the near future as active elements in nonlinear optics and solid state laser applications $\mathrm{s}^{1-3}$. Their small dimensions and rapid growth process make SCFs very interesting for applications in low cost compact solid state devices. However, high optical quality SCF devices must be devoid of diameter fluctuations, which are responsible for light scattering at the fiber/ambient interface ${ }^{3}$. Diameter fluctuations caused by the growth process must be considered as an intrinsic defect of the SCFs, since they are virtually impossible to be corrected afterwards.

Laser Heated Pedestal Growth (LHPG) is one of the most versatile technique for the SCF production. Because it is a meniscus-controlled process, changes in the equilibrium mechanical angle with time introduce diameter fluctuations in the growing fiber. The angle of the meniscus can be modified by any minor imperfections in the pedestal. In this paper, we present an automatic diameter control (ADC) system applied to the growth of SCFs based on an artificial vision apparatus that interacts with the laser power and the pulling rate of the fiber. The use of this system enable us to reduce the diameter fluctuation of the oxide SCFs, grown from unreacted and non-sinterized pedestals, to less than
$2 \%$. The average diameter fluctuations on those SCFs, over its the entire length, could be reduced to less than $1 \%$. Another feature of the control system, is the possibility of causing in situ periodic doping in the growing fiber by modulating the molten zone height. Pure and $\mathrm{Cr}^{3+}$ doped $\mathrm{LaAlO}_{3}$ and pure $\mathrm{LiNbO}_{3}$ were used as model materials.

\section{Automatic Diameter Control and the LHPG System Description}

Our laser heated pedestal growth apparatus was built in its conventional configuration ${ }^{4}$, with an aluminum Reflaxicon $^{5}$ and a spherical focusing mirror. The focusing system is located inside a growth chamber, which allows for airflow to be avoided and for growth to be carried out under different gas atmosphere. The growth chamber has two windows, one of which is used by the crystal grower and the other, located $90^{\circ}$ from the first, where a microscope (Infinity continuous focus microscope) with a CCD camera (Sony, model SSC-C350) attached to it, is used to capture the molten zone image.

Our ADC system uses the measurements on a CCD camera image as a feedback for the $\mathrm{CO}_{2}$ laser (Synrad, model 57-1) and the fiber pulling motor. The video capture board (Interactive technology, model MVLITE) captures the im-

*e-mail: marcello@if.sc.usp.br 
age, which is recorded and processed by a $\mathrm{PC}$ computer (IBM PC - $200 \mathrm{MHz}$ ), in gray scale mode. The black body radiation from the molten zone and the high temperature gradient at the melting (pedestal) and crystallization (fiber) interfaces $\left(10^{3}-10^{4} \mathrm{C} / \mathrm{cm}\right)$, makes it easy to identify these interfaces positions, due to the good contrast in the image at those points. Our software uses those positions to measure the fiber diameter and height of the molten zone.

Two pre-defined areas in the image are measured. The first area is a vertical rectangle $(3 \times 354$ pixels $)$ and the other one is a horizontal rectangle $(354 \times 3$ pixels $)$, as shown in Fig. 1. The software program recognizes the value of all the pixels (from 0 to 255) inside these areas. The next step is to count the number of white pixels inside the pre-define area (molten material). The number of these pixels is proportional to the height of the molten zone (vertical) and to the fiber diameter (horizontal). Based on the vertical pixel count, the software program recognizes the position of the crystallization interface, using this information to take the horizontal measurement just few pixels below the crystallization interface (because of the better contrast on the molten zone). After selecting the desired diameter and molten zone height, the software program modifies the laser power and pulling speed to match the number of pixels established by the crystal grower (set point). In other words, to start the ADC system, the crystal grower needs only to input the number of pixels desired for the diameter and molten zone height, through a graphical interface, and the system will search for this values by changing the laser power and pulling speed.

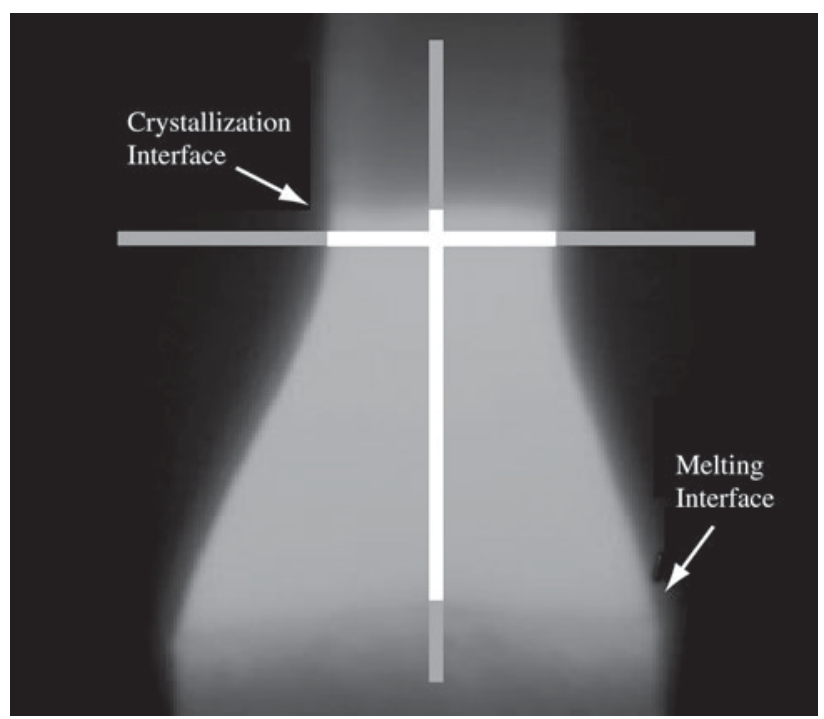

Figure 1. Measurement in a captured image of a $\mathrm{LaAlO}_{3}$ growing fiber.

\section{Pedestal Preparation}

Two different melting point materials were used as a model to verify the versatility of our automatic diameter control system. One compound was $\mathrm{LiNbO}_{3}$ (M. P. $1256^{\circ} \mathrm{C}$ ), whose pedestal for the fiber growth was obtained by cutting bulk single crystals previously grown at our laboratories by Czochralski technique. The pedestals had a squared section of $700 \times 700 \mu \mathrm{m}^{2}$ and length of $3 \mathrm{~cm}$. The other compound used was pure and $\mathrm{Cr}^{3+}$ doped $\mathrm{LaAlO}_{3}$ (M.P. $2110^{\circ} \mathrm{C}$ ) pedestals that were prepared by only mixing the proper amounts of the oxides $\left(\mathrm{Al}_{2} \mathrm{O}_{3}-3 \mathrm{~N}, \mathrm{La}_{2} \mathrm{O}_{3}-2 \mathrm{~N} 5\right.$, $\mathrm{Cr}_{2} \mathrm{O}_{3}-5 \mathrm{~N}$ ), with an organic ligant as described previously by Andreeta et al. ${ }^{6}$. The mixture was then converted into cylindrical pedestal shape, with 1.0 or $1.6 \mathrm{~mm}$ in diameter, using a cold extrusion apparatus, and dried in air for $2 \mathrm{~h}$. The pedestals were not heat treated prior to the growth process itself. Thus, they reacted and sinterized during the growth process as the material approached the molten zone (higher temperature). $\mathrm{LaAlO}_{3}: \mathrm{Cr}^{3+}$ pedestals with a nominal concentration of $0.1 \mathrm{~mol} \%$ were used in the molten zone modulation experiments.

\section{Growth Process}

The growth process began with the melting of the pedestal, after which the seed touched the melt. After waiting 2 min for temperature and molten zone shape to stabilize, the growth process was started. The seed was oriented along the c-axis for lithium niobate growth, while no crystallographic oriented seeds were used in the case of $\mathrm{LaAlO}_{3}$. The growth process was carried out in air atmosphere without seed nor pedestal rotation. The fibers obtained were in the range of 20 to $40 \mathrm{~mm}$ in length. Lithium niobate fibers with diameter of approximately $700 \mu \mathrm{m}$ were obtained using a 1.0 fiber/pedestal pulling ratio. The $700 \mu \mathrm{m}$ and $400 \mu \mathrm{m}$ diameter $\mathrm{LaAlO}_{3}$ fibers were obtained using fiber/pedestal pulling ratios of 0.7 and 1.0 respectively.

For the $\mathrm{LaAlO}_{3}: \mathrm{Cr}^{3+}$ growth process, a periodic fluctuation (sinusoidal function) on the molten zone height was introduced by making continuous changes to the pixel set point. In this way, the molten zone height constantly changed its value. This was done to produce periodic in situ doping of the fiber and to check the effectiveness of the control.

\section{Fiber Characterization}

The SCFs were characterized by optical microscopy and micro-photoluminescence. Optical microscopy was used to measure the diameter fluctuations along the length of the fibers, and to verify that it did not contain any visible inclusions. After the growth process, the $\mathrm{LaAlO}_{3}: \mathrm{Cr}^{3+}$ fibers were fit into a glass support and polished in the axial direction until the center of the fiber was reached. A Raman Renishaw 
Ramanscope 2000 with an automatic micrometric sample translation device and a light pump wavelength of $514.5 \mathrm{~nm}$ (Argon laser) was used to take the micro-photoluminescence measurements along the length of the fibers. We assume that the intensity of the micro-photoluminescence in a particular section of the sample is proportional only to the concentration of dopant at a constant light pump intensity (5 mW).

\section{Results and Discussion}

During the lithium niobate experiments, the molten zone height was measured as a function of the time elapsed in each experiment with and without the molten zone height control activated, and with pulling rate kept equal to zero, Fig. 2. The molten zone height was found to decrease over time when the ADC was not activated. On the other hand, when the ADC was activated, no changes were observed for intervals of over one hour. The average value of the molten zone data, in the measurements with ADC on setup, allowed us to obtain that its height can be controlled within a precision of $30 \mu \mathrm{m}$. The pure $\mathrm{LaAlO}_{3}$ was also pulled with the ADC activated and deactivated. Figure $3 \mathrm{a}$ shows pure $\mathrm{LaAlO}_{3}$ grown with the ADC in the activated mode. Figure $3 \mathrm{~b}$ shows typical diameter measurements as a function of fiber length. Growth was unstable when the ADC was not activated (nominal laser power and pulling rate kept constant), and was common for the molten zone to detach from the fiber, as illustrated in Fig. 3b. This occurs due to the changes in the liquid volume, at the molten zone, during the growth process. The causes for these volume variations are related to the actual laser power and pulling rate fluctuations, even when the nominal values are kept constant, and also to possible imperfections on the pedestal dimensions. Using the ADC device allowed us to reduce fiber diameter fluctuation to less than $2 \%$ of its average diameter as well as to reduce the average diameter fluctuations, to less than $1 \%$ along several millimeters of fiber length during the growth process.

Another experiment was performed to verify the efficacy of the ADC apparatus. Pedestals were prepared with imposed diameter fluctuations up to $20 \%$. Figure 4 illustrates the measurements of the pedestal and fiber diameters as a function of their lengths. The diameter fluctuations obtained were less than $2 \%$, demonstrating the efficiency of the ADC system.

To introduce periodic doping in the fiber during the growth, the molten zone height was modulated with a sinusoidal function, using our ADC system. Fibers were grown with four different oscillation frequencies ranging from $2 \mathrm{mHz}$ to $16 \mathrm{mHz}$. The ADC was activated only a few minutes after rather than at the beginning of the process. Thus, the ADC periodic molten zone oscillation procedures were

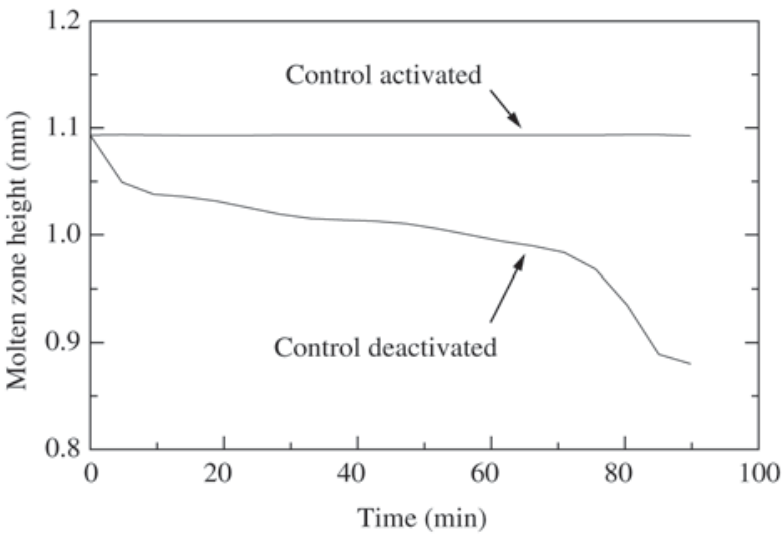

Figure 2. Measurement of the molten zone height in a $\mathrm{LiNbO}_{3}$ fiber growth experiments with the ADC on and off. With ADC off the molten zone height does not keep constant with time.

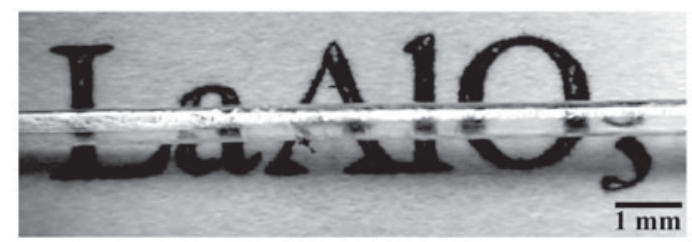

(a)

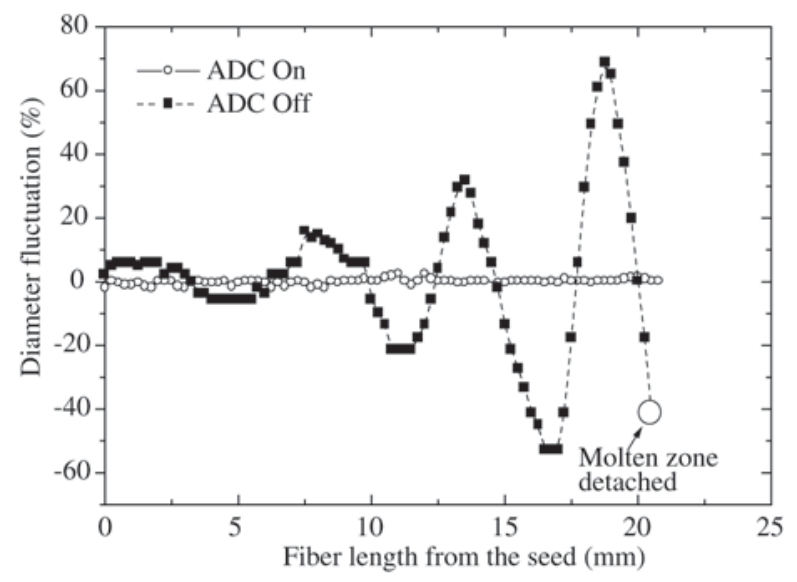

(b)

Figure 3. Diameter fluctuation in $\mathrm{LaAlO}_{3}$ grown fibers. a) Photograph of a fiber grown with ADC on setup; b) Diameter measurements as a function of fiber length for fibers with $700 \mathrm{~mm}$ in diameter. Open dots are for ADC on setup and black dots for a fiber grown without the software control. At the end of the ADC off experiment, the molten zone detached from the growing fiber due to the growth instability. 


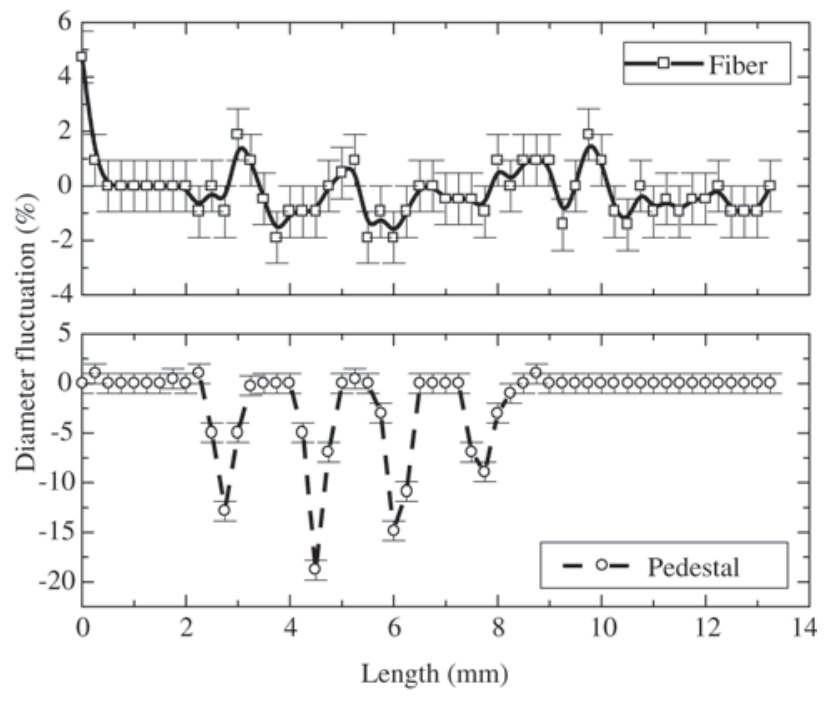

Figure 4. Diameter fluctuation measurements as a function of their lengths for the fiber and pedestal. The pedestal in this case was produced with a diameter fluctuations up to $20 \%$.
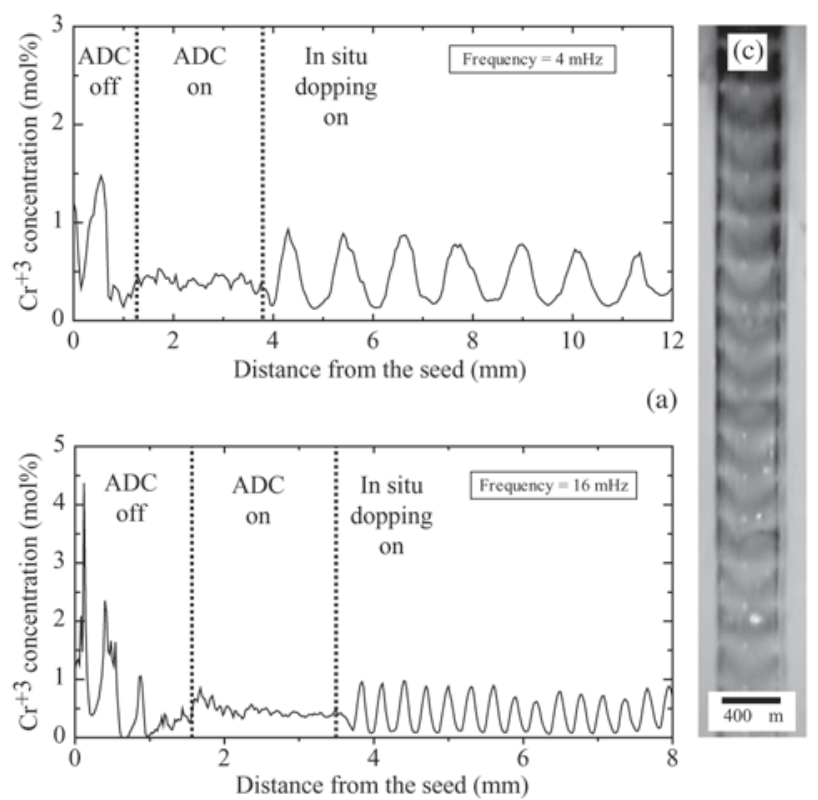

(b)

Figure 5. Axial concentration profile measurement for 2 different molten zone oscillation frequencies. a) frequency $=4 \mathrm{mHz}$; b) frequency $=16 \mathrm{mHz} ; \mathrm{c}$ ) photograph of $\mathrm{LaAlO}_{3}: \mathrm{Cr}^{3+}$ fiber, with periodic axial $\mathrm{Cr}^{3+}$ concentration profile, generated with the molten zone frequency of $16 \mathrm{mHz}$. activated only after the process stabilized. The micro-photoluminescence measurements along the length of the fiber, Fig. 5, clearly indicate that the concentration of the dopant along the fiber fluctuates in a sinusoidal manner when the oscillation of the molten zone is activated, demonstrating the feasibility of in situ axial control of dopant concentration during the fiber growth. We did not find any inclusions inside these fibers, even in the higher concentrations regions. We believe that the reason for this is the low starting $\mathrm{Cr}^{3+}$ concentration used. However, like any other doping process, there should be a concentration limit before dopant inclusions start to occur and the fiber loose its optical quality.

\section{Conclusions}

We have presented an automatic diameter control system (ADC) for the laser heated pedestal growth technique (LHPG), based on an artificial vision apparatus that allows the diameter fluctuation to be reduced to less than $2 \%$, during the growth process of unreacted and non-sinterized pedestals, even when the pedestal diameter fluctuations were of $20 \%$. We have also shown that it is possible to in situ periodic doping of the fiber by modulating the molten zone height with this system. The ADC apparatus allows for control of the axial dopant concentration profile of a SCFs during the growth process, as demonstrate here in the case of $\mathrm{LaAlO}_{3}: \mathrm{Cr}^{3+}$.

\section{Acknowledgements}

The authors would like to thank Dr. Fernando AgullóRueda for the micro-photoluminescence measurements and FAPESP and CNPq (Brazilian research funding institutions) for their financial support.

\section{References}

1. Fejer, M.M.; Nightingale, J.L.; Magel, G.A.; Byer, R.L. Rev. Sci. Instrum., v. 55, p. 1791, 1984.

2. Prokofiev V.V.; Lima, C.J.; Andreeta, M.R.B.; Hernandes, A.C.; Carvalho, J.F.; Andreeta, J.P.; Kamshilin, A.A.; Jaaskelainen, T. J. Crystal Growth, v. 137, p. 528, 1994. 3. Yusheng, D.; Yujing, H.; Zhelin H. Chinese Phys. Lett. 8, v. 12, p. 622, 1991.

4. Fejer, M.M.; Magel, G.A.; Byer, R.L. Applied Optics, v. 24 , n. 15 , p. $2362,1985$.

5. Edmonds, W.R. Appl. Opt. 12, p. 1940, 1973.

6. Andreeta M.R.B.; Hernandes, A.C. J. Crystal Growth, v. 200, n. 3-4, p. 621, 1999. 